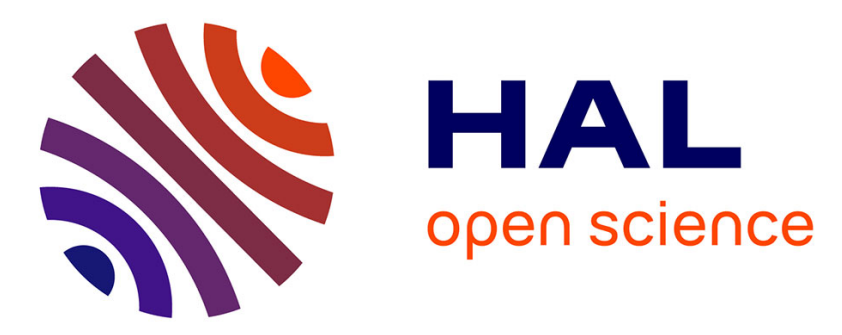

\title{
Reduction and stability analysis of a transcription-translation model of RNA polymerase
}

Ismail Belgacem, Stefano Casagranda, Edith Grac, Delphine Ropers, Jean-Luc Gouzé

\section{- To cite this version:}

Ismail Belgacem, Stefano Casagranda, Edith Grac, Delphine Ropers, Jean-Luc Gouzé. Reduction and stability analysis of a transcription-translation model of RNA polymerase. Bulletin of Mathematical Biology, 2018, 80 (2), pp.294-318. 10.1007/s11538-017-0372-4 . hal-01655367

\section{HAL Id: hal-01655367 https://hal.inria.fr/hal-01655367}

Submitted on 4 Dec 2017

HAL is a multi-disciplinary open access archive for the deposit and dissemination of scientific research documents, whether they are published or not. The documents may come from teaching and research institutions in France or abroad, or from public or private research centers.
L'archive ouverte pluridisciplinaire $\mathbf{H A L}$, est destinée au dépôt et à la diffusion de documents scientifiques de niveau recherche, publiés ou non, émanant des établissements d'enseignement et de recherche français ou étrangers, des laboratoires publics ou privés.

$$
\text { Copyright }
$$




\title{
Reduction and stability analysis of a transcription-translation model of RNA polymerase
}

\author{
Ismail Belgacem · Stefano Casagranda · Edith \\ Grac · Delphine Ropers · Jean-Luc Gouzé
}

Received: date / Accepted: date

\begin{abstract}
The aim of this paper is to analyze the dynamical behavior of biological models of gene transcription and translation. We focus on a particular positive feedback loop governing the synthesis of RNA polymerase, needed for transcribing its own gene. We write a high-dimension model based on mass-action laws and reduce it to a two-variable model (RNA polymerase and its mRNA) by means of monotone system theory and time-scale arguments. We show that the reduced model has either a single globally stable trivial equilibrium in $(0,0)$, or an unstable zero equilibrium and a globally stable positive one. We give generalizations of this model, notably with a variable growth rate. The dynamical behavior of this system can be related to biological observations on the bacterium Escherichia coli.
\end{abstract}

Keywords Biochemical models · Transcription-translation model · Reduction · Stability $\cdot$ Monotone systems

\section{Introduction}

The central dogma of molecular biology argues that "DNA makes RNA and RNA makes proteins", which are the primary components of cells, see [1]. Gene expression starts with transcription, where the gene is copied into a messenger RNA (mRNA) by the RNA polymerase. The mRNA is then translated into proteins by ribosomes. In prokaryotic cells like bacteria, transcription and translation take place in the same compartment. As a consequence, ribosomes can translate nascent mRNAs, while they

Ismail Belgacem, Stefano Casagranda, Jean-Luc Gouzé

Université Côte d'Azur, Inria, INRA, CNRS, UPMC Univ Paris 06, France

Tel.:+33 (0)4 971553 86, +33 (0)4 92387875

E-mail: ismail.belgacem@inria.fr, stefano.casagranda@inria.fr, jean-luc.gouze@inria.fr

Edith Grac, Delphine Ropers

Univ. Grenoble - Alpes, Inria, Grenoble, France

Tel.: +33 (0)4 76615372

E-mail: edith.grac@netcourrier.com,delphine.ropers@inria.fr 
are still being elongated by the RNA polymerase.

Classical models of gene expression often disregard the effect of RNA polymerase and ribosome concentrations on the accumulation of RNAs and proteins, which are assumed non limiting. Recent works have challenged this vision (see [11] for an example), and shown the need for models that take into account the main actors of transcription and translation. Existing models of this type describe some aspects of gene expression and the biogenesis of these cell machineries, see [14,17,23,33] for example. Here we focus on one key mechanism regulating the intracellular levels of RNA polymerase, the auto-regulation of its own synthesis. This process is fundamental for bacterial cells, since the RNA polymerase is encoded from a single gene. This is also an attractive example of positive feedback loop from the point of view of Control Theory.

Based on mass-actions laws, we first write a detailed mechanistic model of transcription and translation, where every event (binding, release, ...) is accounted for. The high dimension of the resulting model makes it too difficult to handle: we reduce it into a much simpler system by time-scale arguments and study the mathematical properties of the reduced model. We investigate the stability of the fast subsystem and of the reduced system by means of monotone system theory and concavity properties. Monotone systems form a class of dynamical systems such that the partial order in dimension $n$ between two solutions is conserved (see [31]); see Appendix A for more details about monotone systems. These tools are well adapted to analyze the stability of biological models [32]. They have strong properties of convergence towards equilibria, and cannot (for example) exhibit stable periodic oscillations. The second tool is related to the concavity of functions used in differential equations [29]. In our opinion, those are appealing tools, for they are qualitative (they do not depend too much on the values of parameters), and they give very strong results about the global dynamical behavior of the system [16]. They have been applied to biological systems already: population dynamics [30], chemical networks [13]... The authors have worked with monotone systems theory on metabolic-genetic networks [5] and on detailed models for gene expression, without any loop [4,6]. In [26], the authors analyze a resource competition model for ribosomes with the theory of monotone systems. However, to the best of our knowledge, the theorem on concave and monotone systems has not been used in the context of detailed gene expression models, where functions can be given by rather complex algebraic expressions resulting from mass balance.

Using monotone system theory, we are able to prove the global stability of the fast sub-system with respect to the equilibrium; the global result is difficult to prove by other techniques, and not proven or proven only locally in most similar works [15, chapter 4]. We present the original model and its reduction in the next section. Using parameters built from the literature, we analyze the different time scales in which evolve the variables of the full system. We decompose the full system into Fast and Slow subsystems and verify in Section 4 that the fast subsystem satisfies the conditions for applicability of the Tikhonov's theorem. This allows us to put the fast subsystem at its quasi-steady state and obtain a reduced model with a similar dynamical behavior. In Section 6, we verify that the concavity and monotonicity assumptions hold for the reduced model. We show that the trivial equilibrium is either globally 


$$
\begin{aligned}
\sigma+P+D & \stackrel{k_{-}}{\leftrightarrows} P D \\
P D & \stackrel{k_{+}}{\rightarrow} Y+D+\sigma \\
Y+N u & \stackrel{k_{t}}{\rightarrow} Y^{1} \\
Y^{1}+N u & \stackrel{k_{t}}{\rightarrow} Y^{2} \\
& \vdots \\
Y^{L-1}+N u & \stackrel{k_{t}}{\rightarrow} P+R N A \\
R N A & \stackrel{k_{m}}{\rightarrow} \phi \\
R+R N A^{\prime} & \stackrel{k_{-}^{\prime}}{\leftrightarrows} R R N A^{\prime} \\
R R N A^{\prime} & \stackrel{k_{+}^{\prime}}{\rightarrow} X+R N A^{\prime} \\
X+t R N A^{*} & \stackrel{k_{\rightarrow}^{\prime}}{\rightarrow} t R N A+X^{1} \\
X^{1}+t R N A^{*} & \stackrel{k_{t}^{\prime}}{\rightarrow} t R N A+X^{2} \\
& \vdots \\
X^{H-1}+t R N A^{*} & \stackrel{k_{t}^{\prime}}{\rightarrow} t R N A+R+P \\
P & \stackrel{k_{p}}{\rightarrow} \phi
\end{aligned}
$$

Fig. 1 Reaction scheme of the transcription-translation model.

stable (in that case no other equilibrium exists) or locally unstable, and that it implies the existence and uniqueness of a positive equilibrium, which is globally stable with respect to the positive orthant. We provide the biological condition for this alternative. We then investigate a generalization of the model with a variable growth rate, compare it with simpler models, and finally give conclusions from a modeling and a biological point of view.

\section{The coupled transcription-translation model of RNA polymerase}

\subsection{Description of the model}

Fig. 1 shows the transcription-translation model for the synthesis of RNA polymerase in a single cell; for simplification, we consider it to be encoded from a single gene. This model is inspired from those given in [23]. Transcription is initiated by the specific binding of RNA polymerase to the promoter region D onto the DNA, a process promoted by an initiating factor called $\sigma$. The RNA polymerase clears the promoter (with a constant rate $k_{c}$ ) and moves along the DNA (with a constant rate $k_{t}$ ). Complexes $Y$ and $Y^{i}$ describe the elongating RNA polymerase, which adds nucleotides one by one. Addition of the last nucleotide completes the full length mRNA, which 
is released from the RNA polymerase. The completed RNA molecule is either subject to degradation (with a constant rate $k_{m}$ ) or it is used by ribosomes as a template for the synthesis of a new RNA polymerase ${ }^{1}$. Translation starts with the ribosome $\mathrm{R}$ forming a complex $R R N A^{\prime}$ with the free ribosome binding site $R N A^{\prime}$ on the newly synthesized mRNA. After clearance of the ribosome binding site (with a constant rate $k_{w}$ ), the elongating form of the ribosome $X$ starts synthesizing the protein: amino acids carried by tRNAs $\left(t R N A^{*}\right)$ are transferred one by one to the nascent protein (with a constant rate $k_{t}^{\prime}$ ), giving the complexes $X^{1}, X^{2}, \ldots, X^{i}$. After addition of the last amino acid, the protein is completed and released by the ribosome. The newly synthesized RNA polymerase is able to start transcribing its own gene and other cellular genes.

The protein and its mRNA are also subject to degradation (with a constant rate $k_{p}$ and $k_{m}$, respectively), and dilution by growth due to the augmentation of cell volume (at a rate $\mu$ ). In a first step, we consider a constant growth rate $\mu$ and, for simplicity, the sum of degradation and dilution will be expressed by only one parameter: $k_{p}^{\prime}$ for the protein and $k_{m}^{\prime}$ for the mRNA. In a second step (Section 9) we will consider the degradation rate and the dilution rate separately, where the latter varies in function of the RNA polymerase concentration.

\subsection{Full equation}

We write the full system, based on assumptions similar to [23]:

- The nucleotides, the amino acids and the $\sigma$ factor are non limiting and their concentrations are included in the parameters

- The free and bound forms of RNA polymerase and mRNA are considered to be degraded at the same rate

- The degradation of the bound forms (PD and $R R N A^{\prime}$ ) releases the promoter and the ribosome

- Free mRNA species correspond to mRNAs with a free ribosome binding site $R N A^{\prime}$.

\footnotetext{
1 The process of translation can be initiated from every nascent mRNA as shown in [23]. For simplicity, we suppose that proteins are synthesized from completed mRNAs only. This is consistent with recent observations on the lack of coupling between transcription and translation in E. coli cells [2].
} 
Using classical mass action kinetics laws we obtain the system:

$$
\begin{aligned}
\dot{c} & =k_{+} p d-k_{-} c-k_{c} c-k_{p}^{\prime} c \\
\dot{d} & =-k_{+} p d+k_{-} c+k_{c} c+k_{p}^{\prime} c \\
\dot{p} & =-k_{+} p d+k_{t} y^{L-1}+k_{-} c+k_{t}^{\prime} x^{H-1}-k_{p}^{\prime} p \\
\dot{y} & =k_{c} c-k_{t} y-k_{p}^{\prime} y \\
\dot{y}^{1} & =k_{t} y-k_{t} y^{1}-k_{p}^{\prime} y^{1} \\
\dot{y}^{2} & =k_{t} y^{1}-k_{t} y^{2}-k_{p}^{\prime} y^{2} \\
\vdots & \\
\dot{y}^{L-1} & =k_{t} y^{L-2}-k_{t} y^{L-1}-k_{p}^{\prime} y^{L-1} \\
\dot{w} & =k_{+}^{\prime} r m-k_{-}^{\prime} w-k_{w} w-k_{m}^{\prime} w \\
\dot{m} & =-k_{+}^{\prime} r m+k_{-}^{\prime} w+k_{w} w+k_{t} y^{L-1}-k_{m}^{\prime} m \\
\dot{r} & =-k_{+}^{\prime} r m+k_{-}^{\prime} w+k_{t}^{\prime} x^{H-1}+k_{m}^{\prime} w \\
\dot{x} & =k_{w} w-k_{t}^{\prime} x \\
\dot{x}^{1} & =k_{t}^{\prime} x-k_{t}^{\prime} x^{1} \\
\vdots & \\
\dot{x}^{H-1} & =k_{t}^{\prime} x^{H-2}-k_{t}^{\prime} x^{H-1}
\end{aligned}
$$

where $p, d, c, y, y^{i}$ and $m$ are the concentrations of $P, D, P D, Y, Y^{i}$ and mRNA respectively, and where $w, r, x$ and $x^{i}$ are the concentrations of $R R N A^{\prime}, R, X$ and $X^{i}$ respectively. $L$ and $H$ are the mRNA and protein lengths, respectively ${ }^{2}$. The promoter $D$ remains intact during the degradation of the RNA polymerase-promoter complex, which means that the following mass conservation relation holds:

$$
d=d_{0}-c
$$

where $d_{0}$ is the initial concentration of promoter. Note that stochasticity in transcription may arise from a small number of molecules (e.g. the low copy number of gene promoters) [25]. However, in this study, we use continuous variables to model the average behavior of RNA polymerase synthesis, within a population of cells. This type of biological information is more easily accessible with experiments on populations of cells than in single cells.

We can reduce system (1) by replacing the three first equations with the following ones:

$$
\begin{aligned}
& \dot{c}=k_{+} p\left(d_{0}-c\right)-k_{-} c-k_{c} c-k_{p}^{\prime} c \\
& \dot{p}=-k_{+} p\left(d_{0}-c\right)+k_{t} y^{L-1}+k_{-} c+k_{t}^{\prime} x^{H-1}-k_{p}^{\prime} p
\end{aligned}
$$

The total number of ribosomes $\left(R_{0}\right)$ is also conserved:

$$
\begin{aligned}
& \frac{d}{d t}\left(r+w+x+x^{1}+\cdots+x^{H-1}\right)=0 \\
& r+w+x+x^{1}+\cdots+x^{H-1}=R_{0}
\end{aligned}
$$

${ }^{2}$ Each triplet of nucleotides in the mRNA are translated into one amino acid, which gives $H=L / 3$. 
Table 1 Values of the set of parameters (fast scale)

\begin{tabular}{llll}
\hline Parameter & Value & Unit & Reference \\
\hline$k_{+}$ & 80 & $\mu M^{-1} \cdot \mathrm{min}^{-1}$ & biologically-reasonable value \\
$k_{-}$ & 40 & $\mathrm{~min}^{-1}$ & biologically-reasonable value \\
$k_{c}$ & 1.5 & $\mathrm{~min}^{-1}$ & {$[12]$} \\
$k_{t}$ & 2340 & nucleotide $\cdot \mathrm{min}^{-1}$ & {$[12]$} \\
$L$ & 8253 & nucleotide & {$[21]$} \\
$k_{+}^{\prime}$ & 11 & $\mu M^{-1} \cdot \mathrm{min}^{-1}$ & biologically-reasonable value \\
$k_{-}^{\prime}$ & 100 & min $^{-1}$ & biologically-reasonable value \\
$k_{w}$ & 55 & min $^{-1}$ & {$[12]$} \\
$k_{t}^{\prime}$ & 1258 & aminoacid $\cdot \mathrm{min}^{-1}$ & {$[12]$} \\
$H$ & 2751 & aminoacid & {$[21]$}
\end{tabular}

Table 2 Values of the set of parameters (slow scale)

\begin{tabular}{llll}
\hline Parameter & Value & Unit & Reference / comment \\
\hline$k_{p}$ & 0.00048 & $\mathrm{~min}^{-1}$ & {$[19]$} \\
$k_{m}$ & 0.17 & $\mathrm{~min}^{-1}$ & {$[10]$} \\
$\mu$ & 0.012 & $\mu M \cdot \mathrm{min}^{-1}$ & {$[12]$} \\
$k_{p}^{\prime}$ & 0.01248 & $\mathrm{~min}^{-1}$ & $k_{p}^{\prime}=k_{p}+\mu$ \\
$k_{m}^{\prime}$ & 0.182 & $\mathrm{~min}^{-1}$ & $k_{m}^{\prime}=k_{m}+\mu$ \\
\hline
\end{tabular}

The above system (1) has a high dimension due to the large size $(L)$ of the mRNA (up to several thousands nucleotides) and of the protein $(H)$, see Table 1 for their values. The system dimension is $L+H+5^{3}$. In addition, the system is non linear and non monotone (for example $\frac{\partial \dot{m}}{\partial r}=-k_{+}^{\prime} m$ is negative), making the analysis of its properties difficult: a simplification of the system is needed in such a case.

\section{Time-scale reduction (Fast-Slow Behavior)}

3.1 Parameter values for the coupled transcription-translation models of RNA polymerase

Parameter values in Tables $1^{4}, 2$ and 3 have been carefully built from the literature based on classical papers such as [12]. In the next section, we will show that the system (1) has two different time scales - fast and slow -, which makes its approximation by a reduced system possible, by applying Tikhonov's Theorem (see Appendix B and [22] for the statement of the theorem).

\footnotetext{
3 The ODE system (1) includes $L$ differential equations for $\dot{y}, \dot{y}^{1} \ldots \dot{y}^{L-1}$ ( $L=8253$ nucleotides), $H$ for $\dot{x}, \dot{x}^{1} \ldots \dot{x}^{H-1}$ ( $L=2751$ amino acids), and 5 for $\dot{c}, \dot{p}, \dot{w}, \dot{m}, \dot{r}$, which gives a total of 11009 differential equations.

${ }^{4}$ The ratios $\frac{L}{k_{t}}$, and $\frac{H}{k_{t}^{\prime}}$ in Table 1 are constant (see [23]). Thus, if we take another gene length $L$ and protein length $H$, the new values of $k_{t}$ and $k_{t}^{\prime}$ respectively to $L$ and $H$ will be $: k_{t}=(2340 / 8253) L$, and $k_{t}^{\prime}=(1258 / 2751) H$. This rescaling is useful to reduce the total number of equations.
} 
Table 3 Initial conditions

\begin{tabular}{llll}
\hline Parameter & Value & Unit & Reference/comment \\
\hline$d_{0}$ & 0.000347 & $\mu M$ & Determined from data in [12] \\
$R_{0}$ & 35 & $\mu M$ & {$[12]$} \\
$z_{0}$ & 0.5 & $\mu M$ & {$[12]$} \\
\hline
\end{tabular}

3.2 Separation of the full system into "fast" and "slow" variables

We choose the following slow variables:

$$
\begin{aligned}
& z=c+p+y+y^{1}+\ldots+y^{L-1} \\
& q=m+w
\end{aligned}
$$

They represent the total concentration (free and bound forms) of the RNA polymerase $z$ and the total concentration of its mRNA $q$. The fast variables appear more easily if some variables are rescaled (see Appendix C). Finally, the full system writes (the slow terms are marked in bold):

$$
\begin{aligned}
\dot{\mathbf{z}} & =\mathbf{k}_{\mathbf{t}}^{\prime} \mathbf{x}^{\mathbf{H}-\mathbf{1}}-\mathbf{k}_{\mathbf{p}}^{\prime} \mathbf{z} \\
\dot{\mathbf{q}} & =\mathbf{k}_{\mathbf{t}} \mathbf{y}^{\mathbf{L}-\mathbf{1}}-\mathbf{k}_{\mathbf{m}}^{\prime} \mathbf{q} \\
\dot{c} & =k_{+} p\left(d_{0}-c\right)-k_{-} c-k_{c} c-\mathbf{k}_{\mathbf{p}}^{\prime} \mathbf{c} \\
\dot{p} & =-k_{+} p\left(d_{0}-c\right)+k_{t} y^{L-1}+k_{-} c+\mathbf{k}_{\mathbf{t}}^{\prime} \mathbf{x}^{\mathbf{H}-\mathbf{1}}-\mathbf{k}_{\mathbf{p}}^{\prime} \mathbf{p} \\
\dot{y} & =k_{c} c-k_{t} y-\mathbf{k}_{\mathbf{p}}^{\prime} \mathbf{y} \\
\dot{y}^{1} & =k_{t} y-k_{t} y^{1}-\mathbf{k}_{\mathbf{p}}^{\prime} \mathbf{y}^{\mathbf{1}} \\
\dot{y}^{2} & =k_{t} y^{1}-k_{t} y^{2}-\mathbf{k}_{\mathbf{p}}^{\prime} \mathbf{y}^{\mathbf{2}} \\
\vdots & \\
\dot{y}^{L-1} & =k_{t} y^{L-2}-k_{t} y^{L-1}-\mathbf{k}_{\mathbf{p}}^{\prime} \mathbf{y}^{\mathbf{L}-\mathbf{1}} \\
\dot{w} & =k_{+}^{\prime} r(q-w)-k_{-}^{\prime} w-k_{w} w-\mathbf{k}_{\mathbf{m}}^{\prime} \mathbf{w} \\
\dot{r} & =-k_{+}^{\prime} r(q-w)+k_{-}^{\prime} w+k_{t}^{\prime} x^{H-1}+\mathbf{k}_{\mathbf{m}}^{\prime} \mathbf{w} \\
\dot{x} & =k_{w} w-k_{t}^{\prime} x \\
\dot{x}^{1} & =k_{t}^{\prime} x-k_{t}^{\prime} x^{1} \\
\vdots & \\
\dot{x}^{H-1} & =k_{t}^{\prime} x^{H-2}-k_{t}^{\prime} x^{H-1}
\end{aligned}
$$

We obtain the fast subsystem by neglecting the bold terms. We group the system in 2 sub-systems. The first one describes the dynamics of the RNA polymerase concentration $z: \dot{z}=k_{t}^{\prime} x^{H-1}-k_{p}^{\prime} z$. The other fast variables $c, p, y, y^{1}, \ldots, y^{L-1}$ have the following dynamics: 


$$
\begin{aligned}
\dot{c} & =k_{+} p\left(d_{0}-c\right)-k_{-} c-k_{c} c \\
\dot{p} & =-k_{+} p\left(d_{0}-c\right)+k_{t} y^{L-1}+k_{-} c \\
\dot{y} & =k_{c} c-k_{t} y \\
\dot{y}^{1} & =k_{t} y-k_{t} y^{1} \\
\dot{y}^{2} & =k_{t} y^{1}-k_{t} y^{2} \\
\vdots & \\
\dot{y}^{L-1} & =k_{t} y^{L-2}-k_{t} y^{L-1}
\end{aligned}
$$

and should leave the hyperplane $z=c+p+y+y^{1}+\ldots+y^{L-1}$ invariant.

The second subsystem describes the dynamics of the mRNA $q$. With $\dot{q}=k_{t} y^{L-1}$ $k_{m}^{\prime} q$, the other fast variables $w, r, x, x^{1}, \ldots, x^{H-1}$ have the following dynamics:

$$
\begin{aligned}
\dot{w} & =k_{+}^{\prime} r(q-w)-k_{-}^{\prime} w-k_{w} w \\
\dot{r} & =-k_{+}^{\prime} r(q-w)+k_{-}^{\prime} w+k_{t}^{\prime} x^{H-1} \\
\dot{x} & =k_{w} w-k_{t}^{\prime} x \\
\dot{x}^{1} & =k_{t}^{\prime} x-k_{t}^{\prime} x^{1} \\
\vdots & \\
\dot{x}^{H-1} & =k_{t}^{\prime} x^{H-2}-k_{t}^{\prime} x^{H-1}
\end{aligned}
$$

and should leave the hyperplane $R_{0}=w+r+x+x^{1}+\ldots+x^{H-1}$ invariant

In Section 4, we will show that the subsystems (5) - with variables $c, p, y, y^{1}, \ldots$, $y^{L-1}$ - and (6) - with variables $w, r, x, x^{1}, x^{L-1}$ - converge to a quasi-steady state, and satisfy the conditions to apply the Tikhonov's theorem and reduce the full system. In particular we will establish that each of the two subsystems has a unique, globally stable equilibrium.

\section{Verification of the applicability of the Tikhonov's Theorem for the fast subsystems}

To check the assumptions of Tikhonov's Theorem, we first need to study the existence and uniqueness of the steady state and the global and exponential stability of the subsystems (5) and (6), representing the fast part of the full system.

When considering the fast subsystem (5), we see that $\dot{c}+\dot{p}+\dot{y}+\dot{y}^{1}+\ldots+\dot{y}^{L-1}=0$ : the system is closed. Powerful theorems apply to this type of monotone system, as can be easily checked: the Jacobian matrix $J\left(c, p, y, y^{1}, \ldots, y^{L-1}\right)$ 


$$
=\left(\begin{array}{ccccc}
-\left(k_{-}+k_{c}-k_{+} p\right) & k_{+}\left(d_{0}-c\right) & 0 & \ldots & 0 \\
k_{-}+k_{+} p & -k_{+}\left(d_{0}-c\right) & 0 & \ldots & k_{t} \\
k_{c} & 0 & -k_{t} & \ldots & 0 \\
0 & 0 & k_{t} & \ldots & 0 \\
\vdots & \vdots & \vdots & \vdots & \vdots \\
0 & 0 & 0 & \ldots & -k_{t}
\end{array}\right)
$$

is a compartmental matrix which means that

$$
\left\{\begin{aligned}
J_{i i}\left(c, p, y, y^{1}, \ldots, y^{L-1}\right) & \leq 0 & & \text { for all } i \\
J_{i j}\left(c, p, y, y^{1}, \ldots, y^{L-1}\right) & \geq 0 & & \text { for all } i \neq j \\
-J_{j j}\left(c, p, y, y^{1}, \ldots, y^{L-1}\right) & \geq \sum_{i \neq j} J_{i j}\left(c, p, y, y^{1}, \ldots, y^{L-1}\right) & & \text { for all } j
\end{aligned}\right.
$$

We can also easily check that the graph of the Jacobian matrix is strongly connected. The interaction graph associated with the Jacobian matrix (7) is shown in Fig. 2 .

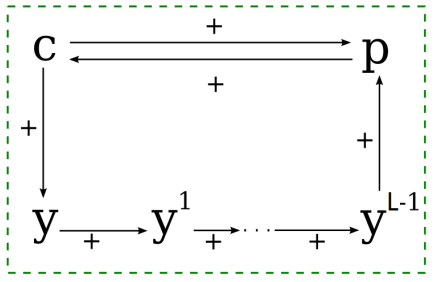

Fig. 2 Interaction graph of system (5).

Therefore, we can apply Property 5 in [3] to obtain the theorem below. The conclusion about exponential stability comes from the fact that the Jacobian matrix at the equilibrium is also a closed compartmental matrix, with a strongly connected graph, and therefore is stable (see $[9,20]$ ) except for the eigenvalue 0 given by the conservation.

Theorem 1 Let $z\left(c, p, y, y^{1}, \ldots, y^{L-1}\right)=c+p+y+y^{1}+\ldots+y^{L-1}$ be the (fixed) total concentration of the closed system.

Then for any $z>0$, hyperplane $H_{z}=\left\{\left(c, p, y, y^{1}, \ldots, y^{L-1}\right) \in \mathfrak{R}_{+}^{L+2}: z\left(c, p, y, y^{1}, \ldots\right.\right.$, $\left.\left.y^{L-1}\right)=z>0\right\}$ is forward invariant and contains a unique equilibrium, globally stable in $H_{z}$.

The proof of this theorem can be obtained using a Lyapunov function (see [20] for more details of this proof ).

The second subsystem (6) is quite similar to subsystem (5). The study of the equilibrium and the stability is exactly the same as before and will not be given here for the 
sake of brevity. We define the hyperplane

$G=\left\{\left(w, r, x, x^{1}, \ldots x^{H-1}\right) \in \mathfrak{R}^{H+2}: w+r+x+x^{1}+\ldots+x^{H-1}=R_{0}\right\}$. The final result is that this system (6) has a unique equilibrium on this invariant hyperplane, which is globally asymptotically stable and exponentially stable.

\section{Application of the Tikhonov's Theorem}

Since the assumptions of the Tikhonov's theorem are verified, we apply it and set the differential equations of the fast part to zero, which gives the following algebraic equations:

$$
\begin{gathered}
y^{L-1}=\ldots=y^{1}=y=\frac{k_{c}}{k_{t}} c=\frac{k_{c} d_{0}}{k_{t}} \frac{p}{p+k_{1}}, \\
x^{H-1}=\ldots=x^{1}=x=\frac{k_{w}}{k_{t}^{\prime}} w=\frac{k_{w}}{k_{t}^{\prime}} \frac{q r}{r+k_{2}}, \\
k_{1}=\frac{k_{-}+k_{c}}{k_{+}}, k_{2}=\frac{k_{-}^{\prime}+k_{w}}{k_{+}^{\prime}} .
\end{gathered}
$$

Moreover, using the two conservation relations on the hyperplanes, and the above algebraic equations, we obtain:

$$
\begin{aligned}
& p+\frac{d_{0} p}{p+k_{1}}+l \frac{k_{c}}{k_{t}} \frac{d_{0} p}{p+k_{1}}=z, \\
& r+\frac{q r}{r+k_{2}}+h \frac{k_{w}}{k_{t}^{\prime}} \frac{q r}{r+k_{2}}=R_{0} .
\end{aligned}
$$

The slow subsystem is always given by

$$
\begin{aligned}
& \dot{z}=k_{t}^{\prime} x^{H-1}-k_{p}^{\prime} z, \\
& \dot{q}=k_{t} y^{L-1}-k_{m}^{\prime} q,
\end{aligned}
$$

which results in the reduced system:

$$
\begin{aligned}
& \dot{z}=k_{w} \frac{q r(q)}{r(q)+k_{2}}-k_{p}^{\prime} z, \\
& \dot{q}=k_{c} d_{0} \frac{p(z)}{p(z)+k_{1}}-k_{m}^{\prime} q .
\end{aligned}
$$

where $p(z)$ and $r(q)$ are calculated from the following algebraic equations:

$$
\begin{aligned}
z & =\gamma \frac{p(z)}{p(z)+k_{1}}+p(z), \\
R_{0} & =\lambda \frac{q r(q)}{r(q)+k_{2}}+r(q) .
\end{aligned}
$$

with $\gamma=\left(L \frac{k_{c}}{k_{t}}+1\right) d_{0}$ and $\lambda=H \frac{k_{w}}{k_{t}^{\prime}}+1$. These algebraic equations have only one positive solution $p(z)$ and $r(q)$, because for fixed $z$ and $q$, the right members of (12) are increasing functions of $p$ and $r$. 


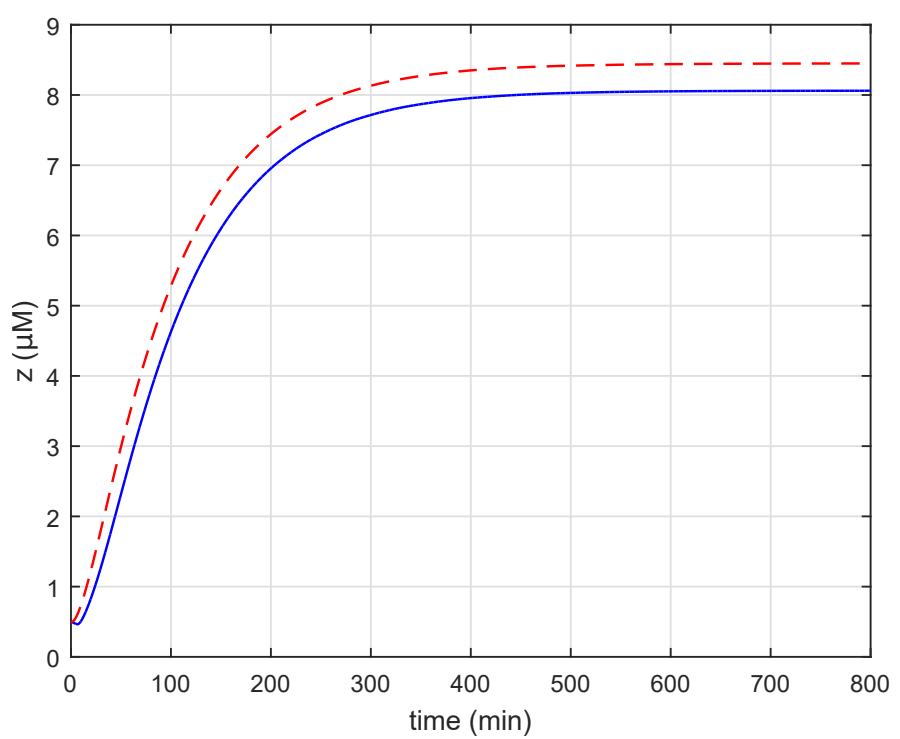

Fig. 3 Time behavior of the total concentration of RNA polymerase $z$ in the reduced system (dashed red line) and in the complete system (solid blue line), with initial conditions: $p_{0}=z_{0}=0.5 \mu M$, taking $L=l=100$ nucleotides, $H=h=33$ amino acids. At the equilibrium: $z_{\text {reduced }}^{*}=8.449 \mu M, z_{\text {full }}^{*}=8.061$ $\mu M$.

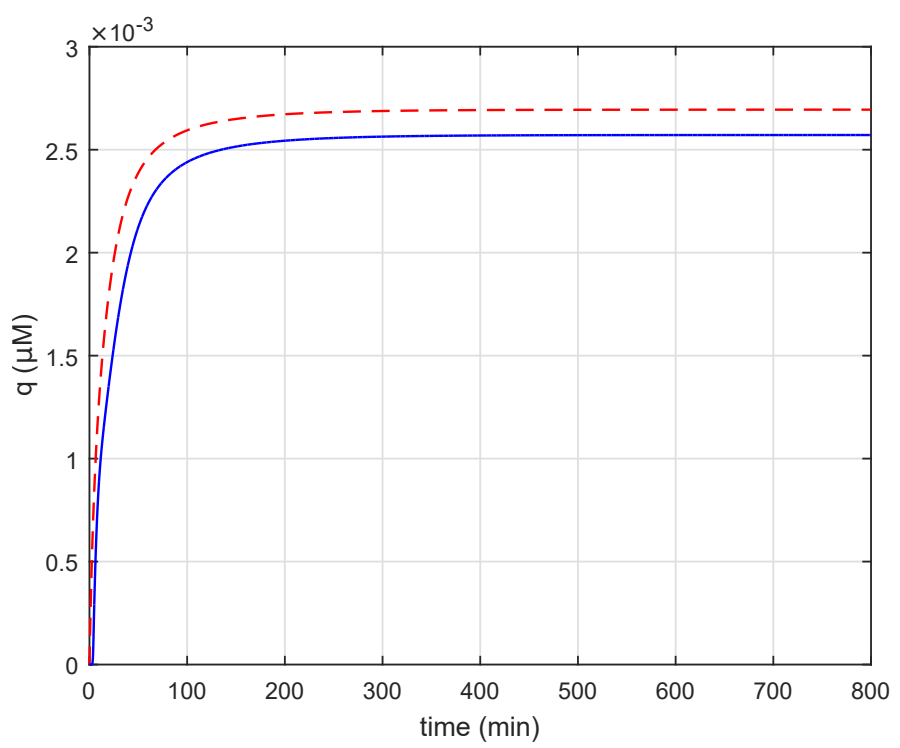

Fig. 4 Time behavior of the concentration of mRNA $q$ in the reduced system (dashed red line) and in the complete system (solid blue line), with $q_{0}=0 \mu M, l=100$ nucleotides, $h=33$ amino acids. At the equilibrium: $q_{\text {reduced }}^{*}=0.002694 \mu M, q_{\text {full }}^{*}=0.002571 \mu M$. 
Note that the Tikhonov's theorem, in its simplest form, gives only results for a compact interval of time. Extensions are possible for infinite time (see [18,22]). For example, if the reduced system has an hyperbolically stable equilibrium point $z$, the full system will admit an hyperbolic equilibrium close to $z$ for small $\varepsilon$. Stronger results can be obtained with geometric perturbation theory (see [27,35]). In particular, in [35], the authors show that if the full system has a slow, monotone part (and if $\varepsilon$ is small enough), almost all trajectories of the full system converge to an equilibrium, which is the general property of monotone systems.

\section{Dynamical study of the reduced system}

6.1 Simulations of the full and the reduced system

We performed simulations ${ }^{5}$ to study the similarity of the reduced system and the full system, as shown in Fig. 3 and Fig. 4 for the concentration of RNA polymerase and its mRNA, respectively. The dashed red line represents the evolution of the reduced system, and the solid blue line shows the evolution of the complete system. The behavior of the two systems are rather similar, the full system slightly oscillates at the beginning, while the reduced system does not.

\subsection{Equilibria of the reduced system}

We get the following equations for the equilibrium:

$$
\begin{aligned}
& z=\frac{k_{w}}{k_{p}} \frac{q r(q)}{r(q)+k_{2}}=\frac{k_{w}}{k_{p}^{\prime} \lambda}\left(R_{0}-r(q)\right), \\
& q=\frac{k_{c} d_{0}}{k_{m}} \frac{p(z)}{p(z)+k_{1}}=\frac{k_{c} d_{0}}{k_{m}^{\prime} \gamma}(z-p(z)) .
\end{aligned}
$$

We therefore obtain $z$ as a function of $q$, and $q$ as a function of $z$. Consider the functions:

$$
\begin{aligned}
\xi(q) & =\frac{k_{w}}{k_{p}^{\prime}} \frac{q r(q)}{r(q)+k_{2}}, \\
\varphi(z) & =\frac{k_{c} d_{0}}{k_{m}^{\prime}} \frac{p(z)}{p(z)+k_{1}} .
\end{aligned}
$$

We first note that $\varphi(z)$ is bounded $\left(\varphi(z)<\frac{k_{c} d_{0}}{k_{m}^{\prime}}\right)$, because $\frac{p(z)}{p(z)+k_{1}}<1$. Similarly, $r(q)=R_{0}-\frac{k_{p}^{\prime} \lambda}{k_{w}} \xi(q)$ should remain positive, which leads to $\xi(q)<\frac{k_{w} R_{0}}{k_{p}^{\prime} \lambda}$. Determining the equilibria requires to study the intersections of the two above functions $\xi(q)$ and $\varphi(z)$. If we differentiate the first equation with respect to $z$ in the algebraic system (12), and the second with respect to $q$, we obtain:

\footnotetext{
5 Numerical simulations were performed in Matlab using the ode15s and ode23s solvers.
} 


$$
\begin{aligned}
& \frac{\partial p(z)}{\partial z}=\frac{\left(p(z)+k_{1}\right)^{2}}{\gamma k_{1}+\left(p(z)+k_{1}\right)^{2}}, \\
& \frac{\partial r(q)}{\partial q}=-\frac{\lambda \frac{r(q)}{r(q)+k_{2}}}{\lambda q \frac{k_{2}}{\left(r(q)+k_{2}\right)^{2}}+1} .
\end{aligned}
$$

$p(z)$ is positive and increasing, with $p(0)=0$ and $p(z) \approx z$ when $z$ is large. Similarly, $r(q)$ is positive and decreasing, with $r(0)=R_{0}$ and $r(q)$ tends toward 0 for large $q$. We then determine the second derivative of the algebraic equations (12), the first equation derived with respect to $z$, and the second, with respect to $q$, and obtain:

$$
\begin{aligned}
\frac{\partial^{2} p(z)}{\partial^{2} z} & =\gamma \frac{2 k_{1}}{\left(p(z)+k_{1}\right)^{3}} \frac{\partial p(z)^{2}}{\partial z}, \\
\frac{\partial^{2} r(q)}{\partial^{2} q} & =\frac{-2 \lambda \frac{k_{2}}{\left(r(q)+k_{2}\right)^{2}} \frac{\partial r(q)}{\partial q}+\lambda q \frac{2 k_{2}}{\left(r(q)+k_{2}\right)^{3}} \frac{\partial r(q)}{\partial q}^{2}}{1+\lambda q \frac{k_{2}}{\left(r(q)+k_{2}\right)^{2}}} .
\end{aligned}
$$

$\frac{\partial^{2} p(z)}{\partial^{2} z}$ and $\frac{\partial^{2} r(q)}{\partial^{2} q}$ are thus positive. We conclude that $p(z)$ and $r(q)$ are also convex. The derivative of $\xi(q)$ is $\xi^{\prime}(q)=-\frac{k_{w}}{k_{p}^{\prime} \lambda} \frac{\partial r(q)}{\partial q}$, which is positive because $\frac{\partial r(q)}{\partial q}$ is negative. We have also that $\xi^{\prime \prime}(q)=-\frac{k_{w}}{k_{p}^{\prime} \lambda} \frac{\partial^{2} r(q)}{\partial q^{2}}$ is negative, because $\frac{\partial^{2} r(q)}{\partial q^{2}}$ is positive. Similarly, for $\varphi(z)$, we have $\varphi^{\prime}(z)=\frac{k_{c} d_{0}}{k_{m}^{\prime}} \frac{k_{1}}{\left(p(z)+k_{1}\right)^{2}} \frac{\partial p(z)}{\partial z}=\frac{k_{c} d_{0}}{k_{m} \gamma}\left(1-\frac{\partial p(z)}{\partial z}\right)$ which is positive, because $\frac{\partial p(z)}{\partial z}$ is positive, and $\varphi^{\prime \prime}(z)=-\frac{k_{c} d_{0}}{k_{m}^{\prime} \gamma} \frac{\partial^{2} p(z)}{\partial z^{2}}$ negative, because $\frac{\partial p(z)}{\partial^{2} z}$ is positive.

The functions $\xi(q)$ and $\varphi(z)$ are therefore increasing, positive and concave, and are bounded $\left(\xi(q)<\frac{k_{w} R_{0}}{k_{p} \lambda}, \varphi(z)<\frac{k_{c} d_{0}}{k_{m}^{\prime}}\right)$.

In the phase space $(q, z)$, two cases are possible, see Fig. 5 and Fig. 6: either $(0,0)$ is the unique equilibrium, or there exists another unique, positive equilibrium (the point $(0,0)$ is always an equilibrium for this system). The alternative between these two cases depends on the slope at the origin $(0,0)$.

- If: $\left.\xi^{\prime}(q)\right|_{q=0}>\frac{1}{\left.\varphi^{\prime}(z)\right|_{z=0}} \Rightarrow k_{m}^{\prime} k_{p}^{\prime}<k_{w} \frac{k_{c} d_{0}}{\gamma+k_{1}} \frac{R_{0}}{R_{0}+k_{2}}$, then there exist two equilibria which are $(0,0)$, and a unique, positive $\left(z^{*}, q^{*}\right)$.

- If: $\left.\xi^{\prime}(q)\right|_{q=0}<\frac{1}{\left.\varphi^{\prime}(z)\right|_{z=0}} \Rightarrow k_{m}^{\prime} k_{p}^{\prime}>k_{w} \frac{k_{c} d_{0}}{\gamma+k_{1}} \frac{R_{0}}{R_{0}+k_{2}}$, then there exists only one equilibrium for the system, which is $(0,0)$.

\subsection{Stability of equilibria}

The stability study of the reduced system (11) gives the following results: it has either a single stable equilibrium in $(0,0)$ or two equilibria, one in zero (unstable) and another stable one $\left(z^{*}, q^{*}\right)$. 


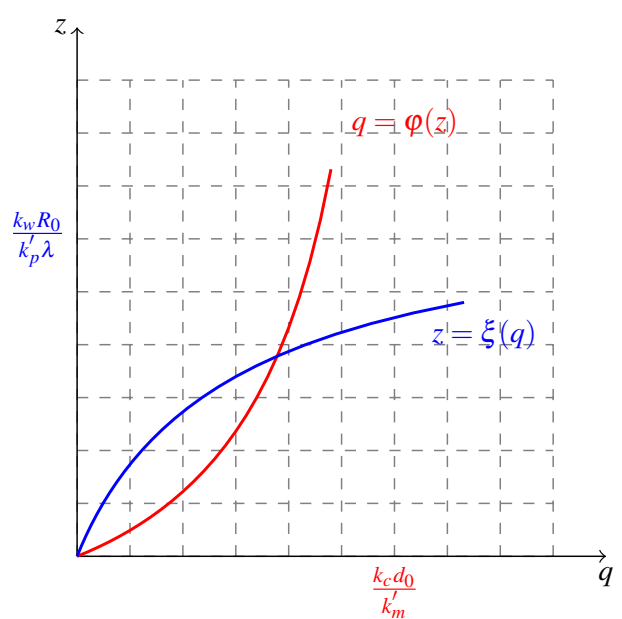

Fig. 5 The phase space $(q, z)$. Two equilibria exist: $(0,0)$ and another one, which is strictly positive $\left(z^{*}, q^{*}\right)$ under the condition $\left.\xi^{\prime}(q)\right|_{q=0}>\frac{1}{\left.\varphi^{\prime}(z)\right|_{z=0}}$.

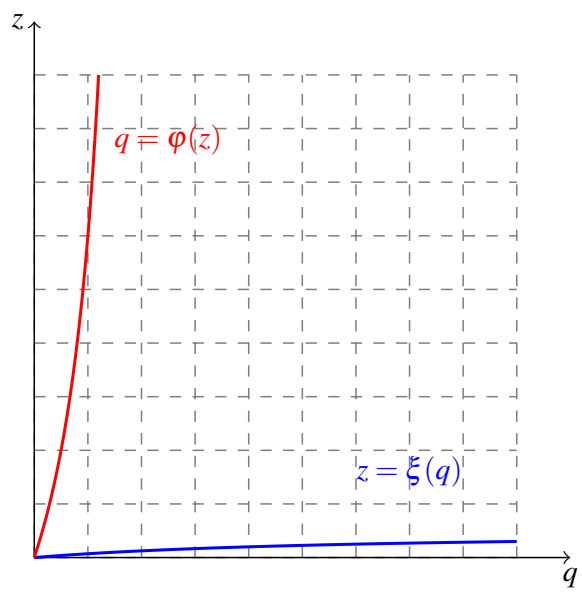

Fig. 6 The phase space $(q, z)$. One equilibrium exists, which is $(0,0)$ if $\left.\xi^{\prime}(q)\right|_{q=0}<\frac{1}{\left.\varphi^{\prime}(z)\right|_{z=0}}$.

Proposition 1 If $k_{m}^{\prime} k_{p}^{\prime}-k_{w} \frac{k_{c} d_{0}}{\gamma+k_{1}} \frac{R_{0}}{R_{0}+k_{2}}>0$ then

- $(0,0)$ is the unique equilibrium of the system and it is globally stable in the nonnegative orthant.

Proposition 2 If $k_{m}^{\prime} k_{p}^{\prime}-k_{w} \frac{k_{c} d_{0}}{\gamma+k_{1}} \frac{R_{0}}{R_{0}+k_{2}}<0$ then

- $(0,0)$ is unstable.

- The positive equilibrium $\left(z^{*}, q^{*}\right)$ is globally stable in the positive orthant. 


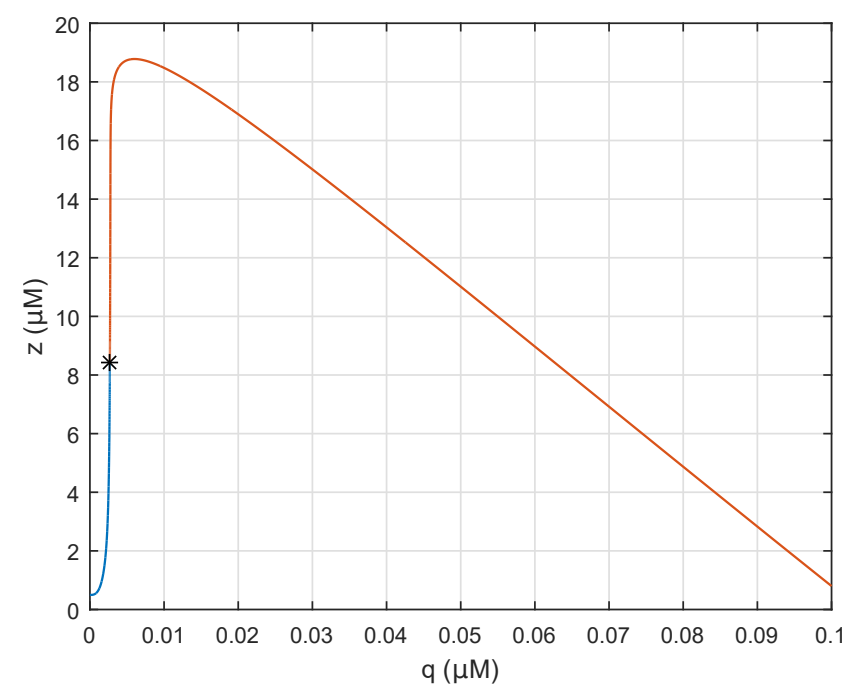

Fig. 7 Simulation with the parameter values in Tables 1 and 2 from two different initial conditions: $\left(q_{01}, z_{01}\right)=(0,0.5)$ (blue line) and $\left(q_{02}, z_{02}\right)=(0.1,0.8)$ (red line). This corresponds to the case that two equilibria exist, $(0,0)$ and $(0.002694,8.449)$ (black star). The latter is globally stable in the positive orthant.

For the proof of these propositions, we use the fact that the system (11) is still monotone [31], see [8] for more details. The biological interpretations of Propositions 1 and 2 are presented in the conclusion.

Fig. 7 shows the simulation of the reduced system from two different initial conditions, $\left(q_{01}, z_{01}\right)=(0,0.5)$ and $\left(q_{02}, z_{02}\right)=(0.1,0.8)$. We observe that the two trajectories converge to the positive equilibrium.

The power of this approach is that it allows results from qualitative hypotheses, even in high dimension. Being qualitative, it can be easily applied to similar models with other assumptions. We consider below the case of different degradation rates.

\section{Applications to other models}

In the above model, we supposed that all forms of RNA polymerase are degraded at the same rate. This assumption could be changed, for example by supposing a degradation of the free form of the polymerase only, but no degradation for the bound 
forms. The new equations are:

$$
\begin{aligned}
z & =\gamma \frac{p}{p+k_{1}}+p, \\
R_{0} & =\lambda \frac{q r}{r+k_{2}}+r, \\
\dot{z} & =k_{w} \frac{q r(q)}{r(q)+k_{2}}-k_{p}^{\prime} p(z), \\
\dot{q} & =k_{c} d_{0} \frac{p(z)}{p(z)+k_{1}}-k_{m}^{\prime}\left(q-\frac{q r(q)}{r(q)+k_{2}}\right) .
\end{aligned}
$$

The conservation equations are the same, but the diagonal elements of the Jacobian matrix change: however, it is easy to verify that our approach is still applicable, and, similarly, the global stability now depends only on the local stability at $(0,0)$ with the new inequality (for the trivial equilibrium) $k_{p}^{\prime} k_{m}^{\prime}>k_{w} \frac{k_{c} d_{0} R_{0}}{k_{1} k_{2}}$. Other generalizations with qualitative functions of Michaelis-Menten type $(M(p)$ is strictly increasing, concave, bounded, and such that $M(0)=0$ ) are possible (see [7], [8]).

\section{Comparison with a classical model of RNA polymerase}

It is interesting to compare the new model (11) with the more "classical" model used to describe gene expression in [1, chapter 2, p. 13], in the case of a protein activating its own transcription:

$$
\dot{\chi}=v_{c} \frac{\chi}{\chi+k_{c}}-\left(k_{p c}+\mu\right) \chi
$$

where $\chi$ is the protein concentration and $\mu$ is the same growth rate as in Table 2. We first substitute in the model (17) $k_{p}^{\prime}$ with $k_{p}+\mu$ and $k_{m}^{\prime}$ with $k_{m}+\mu$. The dilution term (term with $\mu$ ) of the equation $\dot{q}$ can be neglected because biological evidences demonstrate that $\mu \ll k_{m}$. To simplify our study, we assume that the dynamics of $\dot{z}$ is much slower than the one of $\dot{q}$, so that $\dot{q} \approx 0$ (by a new quasi-steady state approximation): in fact most of the time mRNA dynamics in genetic regulatory networks is much faster than protein dynamics, i.e. the mRNA concentration reaches its equilibrium faster than that of the protein (typical mRNA half-lives are 2-6 minutes, while those of proteins are in the order of hours [1]). With this assumption, we obtain system (19):

$$
\begin{aligned}
\dot{z} & =k_{w} \frac{q r(q)}{r(q)+k_{2}}-\left(k_{p}+\mu\right) z \\
q & =\frac{k_{c} d_{0}}{k_{m}} \frac{p(z)}{p(z)+k_{1}} .
\end{aligned}
$$

We use the points of the solution $z(t)$ to fit the model $(18)^{6}$ and find the values of the parameters $v_{c}, k_{c}, k_{p c}$, shown in Table 4 , so that the dynamical behavior of the solution $\chi(t)$ is the closest to the one of $z(t)$, where $\chi(0)=z(0)$. To assess the qual-

6 The fit was performed using the fmincon function of Matlab. We thank Eugenio Cinquemani (Inria, IBIS) for his help with the optimization procedure. 
Table 4 Parameters of $\chi(t)$

\begin{tabular}{lll}
\hline Parameter & Value & Unit \\
\hline$v_{c}$ & 0.1251 & $\mu M \cdot \mathrm{min}^{-1}$ \\
$k_{c}$ & 0.6452 & $\mu M$ \\
$k_{p c}$ & 0.00087264 & $\mathrm{~min}^{-1}$ \\
\hline
\end{tabular}

ity of the calibration, we numerically compute the global relative error between the solution $z(t)$ and the system $\chi(t)$. One possible form of global relative error is:

$$
e_{\chi}=\frac{\int_{0}^{T}|z(t)-\chi(t)| d t}{\int_{0}^{T}|z(t)| d t},
$$

where $T=800 \mathrm{~min}$. With the parameter values of Table 4 , the relative global error is low ( $e=0.0016$ ). This means that the two solutions are very close: the model (19) can fit simpler classical models.

However, we can observe phenomena of biological interest with our model that are not captured by the "classical" model, especially in the translation term. If, in (19), we substitute the algebraic expression for $q$ in the differential expression of $z$ and isolate the translation term, we have:

$$
\tau=k_{w} \frac{q r(q)}{r(q)+k_{2}} .
$$

Contrary to the "classical" one, this model shows that the translation rate $\tau$ is sensitive to the ribosome concentration $R_{0}$, due to the dependence between $r(q)$ and $R_{0}$ (for $q=0, r(0)=R_{0}$ ) in the algebraic part of system (17). This is reflected in Fig. 8, which displays the translation rate $\tau$ as a function of the RNA polymerase concentration, for different concentrations of $R_{0}$. Increasing ribosome concentrations directly result in elevated translation of RNA polymerase. The three upper curves represent systems following the case of Proposition 1 (one positive stable equilibrium) and the lower curve, the case of Proposition 2 ( 0 is the only stable equilibrium). Obviously, these different curves cannot be generated by the simpler model (18).

\section{System with a variable growth rate}

In the above sections, we considered a fixed growth rate for the model (11). In the reality, bacteria experience a variable growth rate most of the time. What is the consequence of this variation on the model dynamics? We consider a one-dimensional model for simplicity, as in Section 8. We study the effect of introducing a variable growth rate, which depends on the concentration of the cell components, here the concentration of RNA polymerase $z$. The growth rate can be expressed by means of a function analogous to the widely used Monod equation in growth kinetic studies [24, p. 211]:

$$
\mu=v \frac{z}{K+z} .
$$




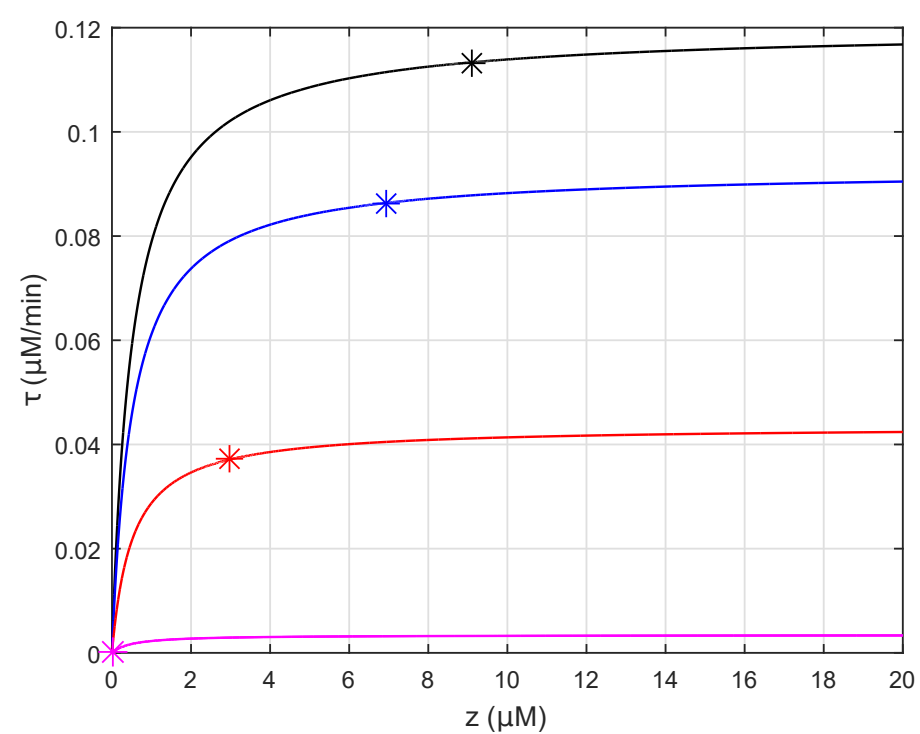

Fig. 8 Variation of the translation rate $\tau$ with the RNA polymerase concentration $z$, for different ribosome concentrations: $R_{0}=35 \mu M$ (black line; in this case the dynamics of $z$ and $\chi$ overlap), $R_{0}=12.5 \mu M$ (blue line), $R_{0}=5 \mu M$ (red line), and $R_{0}=0.3 \mu M$ (magenta line). The star in each curve denotes the equilibrium points, respectively $z=9.082 \mu M, z=6.919 \mu M, z=2.972 \mu M, z=0 \mu M$.

Table 5 Growth Rate Parameters

\begin{tabular}{lll}
\hline Parameter & Value & Unit \\
\hline$K$ & 8.85 & $\mu \mathrm{M}$ \\
$v$ & 0.0237 & $\mu \mathrm{M} \cdot \mathrm{min}^{-1}$ \\
\hline
\end{tabular}

The previous system (19) becomes:

$$
\begin{aligned}
& \dot{z}=k_{w} \frac{q r(q)}{r(q)+k_{2}}-\left(k_{p}+v \frac{z}{K+z}\right) z, \\
& q=\frac{k_{c} d_{0}}{k_{m}} \frac{p(z)}{p(z)+k_{1}} .
\end{aligned}
$$

A way to find reasonable values for parameters $v$ and $K$ is to substitute, in Equation (22), the equilibrium point that the variable $z$ reaches in system (19), $z^{*}$, to obtain

$$
\mu\left(z^{*}\right)=v \frac{z^{*}}{K+z^{*}} .
$$

We manually set parameter values in Table 2 so that $\mu\left(z^{*}\right)$ reaches the value of the constant $\mu$ in Table 2 and that system (23) reaches the same equilibrium point as system (19). The simulation for $z$ is shown in Fig. 9 and the growth rate dynamics, in Fig. 10. 


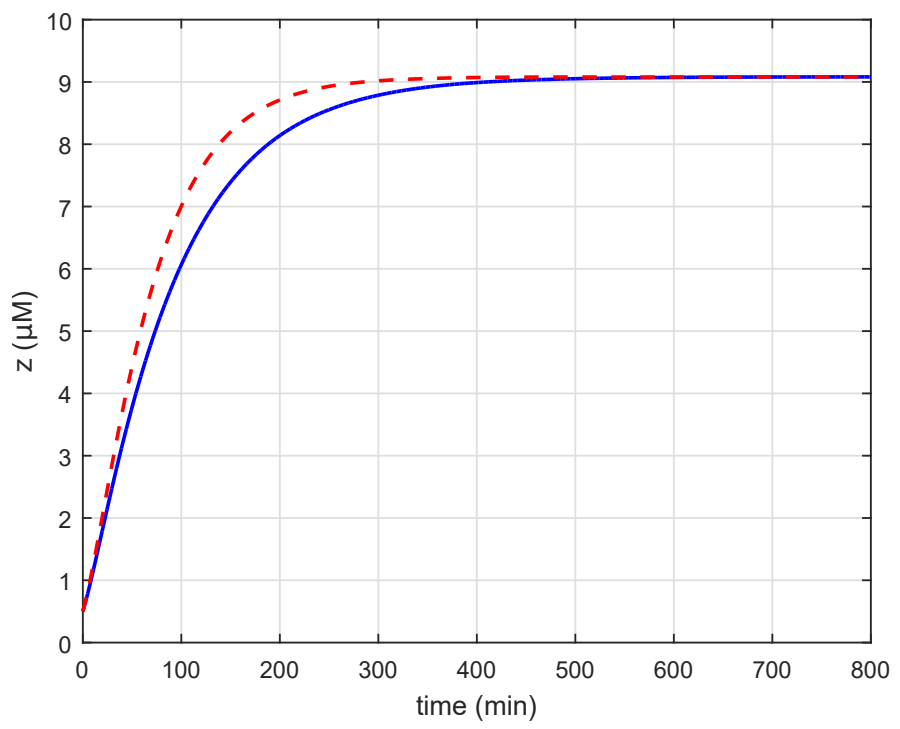

Fig. 9 Evolution of the total concentration of RNA polymerase $z$ in the case of a dependent growth rate (dashed red line), compared with system (19) (solid blue line). Initial conditions are: $p_{0}=z_{0}=0.5 \mu M$, $L=l=100$ nucleotides and $H=h=33$ amino acids. The final equilibrium is the same at $9.084 \mu M$

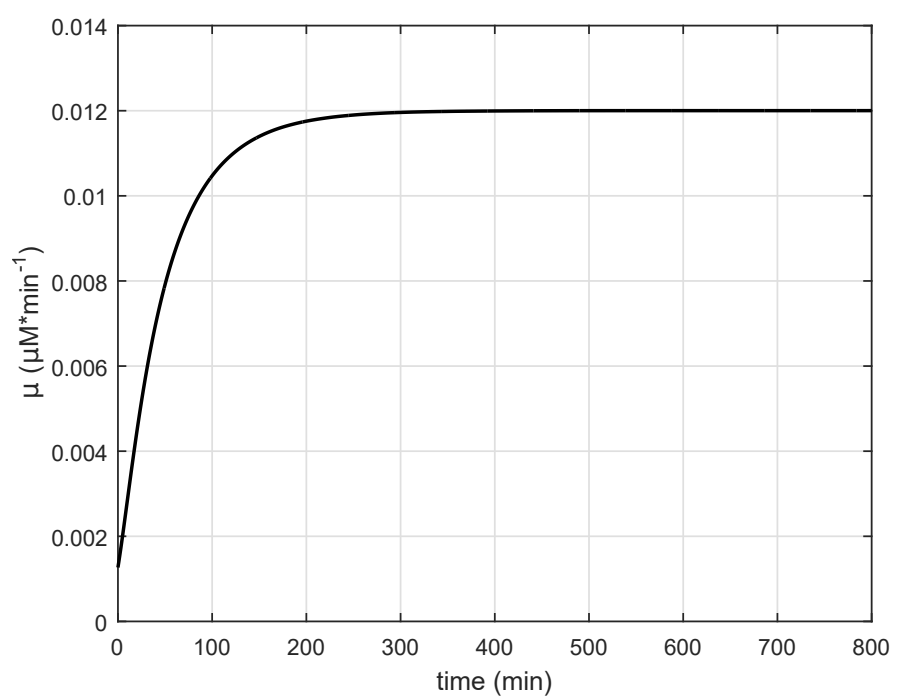

Fig. 10 Growth rate dynamics with the parameters of Table 5. 
To analyze the new system, we notice that the first term in the equation of $\dot{z}, G(z)=$ $H(q(z))=k_{w} \frac{q(z) r(q(z))}{r(q(z))+k_{2}}$, is a function of $q$, which in turn is a function of $z$. Using the same notation as (14): $G(z)=\xi(\varphi(z))$, the derivative of this function is $G^{\prime}(z)=$ $\xi^{\prime}(\varphi(z)) \varphi^{\prime}(z)$. Both terms are positive (as discussed in Section 6), so the derivative is positive. The second derivative is $G^{\prime \prime}(z)=\xi^{\prime \prime}(\varphi(z)) \varphi^{\prime}(z) \varphi^{\prime}(z)+\xi^{\prime}(\varphi(z)) \varphi^{\prime \prime}(z)$. The first and last terms are negative and the others, positive (as we discussed in Section 8), so the second derivative of the function is negative. $G(z)$ is therefore increasing, positive, concave and it is bounded $\left(\frac{k_{c} d_{0} k_{w}}{k_{m}}\right)$. The second term of the equation $D(z)=k_{p} z+v \frac{z^{2}}{K+z}$ is positive, increasing and convex: depending on the values of the parameters, the functions $G(z)$ and $D(z)$ can have one or two equilibrium points:

- If : $\left.D^{\prime}(z)\right|_{z=0}<\left.G^{\prime}(z)\right|_{z=0} \Rightarrow k_{m} k_{p}<k_{w} \frac{k_{c} d_{0}}{\gamma+k_{1}} \frac{R_{0}}{R_{0}+k_{2}}$, there exist two equilibria which is 0 , and a unique, positive $z^{*}$

- If: $\left.D^{\prime}(z)\right|_{z=0}>\left.G^{\prime}(z)\right|_{z=0} \Rightarrow k_{m} k_{p}>k_{w} \frac{k_{c} d_{0}}{\gamma+k_{1}} \frac{R_{0}}{R_{0}+k_{2}}$, there exists only one equilibrium for the system which is 0 .

Following the same rules as in Section 6.3, we see that in the first case the equilibrium point $z^{*}$ is globally stable and 0 is globally unstable, while in the second case 0 is globally stable.

We notice that the growth rate, which depends only on the concentration of RNA polymerase $z$, does not play any role in the qualitative stability of the system. Of course, it changes the trajectories, as shown in Fig 9.

\section{Conclusion}

Several interesting conclusions can be drawn from this study. First, we demonstrate that tools from monotone theory are useful for proving stability of the fast and slow parts of a system. We showed that the positive feedback loop of the polymerase model that could, a priori, give a bistable behaviour, will result in this case in a simple "global behaviour" where the trivial equilibrium (if no other equilibrium) is stable, or the positive equilibrium (when it exists) is stable. Moreover, biological conclusions can be drawn. For example, computations lead to the fact that the system is stable depending on the sign of (see Proposition 1 and Proposition 2):

$$
k_{m} k_{p}-k_{w} \frac{k_{c} d_{0}}{\gamma+k_{1}} \frac{R_{0}}{R_{0}+k_{2}} .
$$

If $R_{0}$ is large (many ribosomes), the zero equilibrium is unstable; if $R_{0}$ is small, the zero equilibrium is globally stable, and every variable tends to zero. These results are in agreement with several biological observations on the adaptation of living organisms to their environment. For instance, in the case of bacteria, the zero equilibrium corresponds to the situation of cells whose growth is arrested by harmful environmental conditions. Translation is halted in these cells, through an arrest of ribosome synthesis and the inactivation of the remaining ribosomes [28,34]. As a consequence, 
the intracellular concentration of active ribosomes decreases, which lowers the concentration of RNA polymerase. The essential cell components can no longer be synthesized; cells eventually die if the ribosomes and the RNA polymerase remains at so low concentrations. By contrast, when environmental conditions become favorable again, ribosome synthesis starts immediately and inactivated ribosomes become functional again $[28,34]$. The intracellular concentration of ribosomes rises. According to the model, the zero equilibrium becomes unstable in these conditions. The consequence is a rapid accumulation of new pools of RNA polymerase and ribosomes, that are necessary for the cell to synthesize all the precursors needed to grow and divide again. Note that this very simple loop is not isolated from the rest of the cell. The simple model could be easily extended so as to include these regulatory mechanisms. As well, the reduced system could be included into more general models of the gene expression machinery.

Adding to the system a variable growth rate (depending only on the RNA polymerase concentration) does not change the stability of the curve, because for $z=0 \Rightarrow$ $\mu^{\prime}(0)=0$. We can reach the same equilibrium point of $z$ with a constant or a variable growth rate as shown in Fig. 9, if $\mu\left(z^{*}\right)=\mu$ of Table 2.

Moreover, it is important to notice that, in restricted cases, the dynamics of our model overlaps with a simpler dynamical model (see Section 8): this confirms the fact that we can explain the dynamics of RNA polymerase in a more precise way, detailing also the relationship with mRNA, without changing its performance with respect to more simpler and classical models.

Finally, an exciting perspective on which we are working is to add a control on this system, for example via the action of an inducer, which activates the transcription step.

Acknowledgements We acknowledge Conseil Régional PACA for funding the $\mathrm{PhD}$ thesis of S.Casagranda. We acknowledge also the Investissements d'Avenir Bio-informatique programme under project RESET (ANR-11-BINF-0005) for funding team IBIS and the PhD thesis of S.Casagranda. We thank the research program Labex SIGNALIFE. The authors wish to thank the anonymous reviewers for their very pertinent remarks.

\section{References}

1. Alon, U.: An Introduction to Systems Biology. Chapman \& Hall/CRC, Boca Raton (2007)

2. Bakshi, S., Siryaporn, A., Goulian, M., Weisshaar, J.C.: Superresolution imaging of ribosomes and RNA polymerase in live Escherichia coli cells. Molecular Microbiology 85(1), 21-38 (2012)

3. Bastin, G., Guffens, V.: Congestion control in compartmental network systems. Systems \& Control Letters 55(8), 689-696 (2006)

4. Belgacem, I., Gouzé, J.L.: Analysis and reduction of transcription translation coupled models for gene expression. IFAC Proceedings Volumes 46(31), 36-41 (2013)

5. Belgacem, I., Gouzé, J.L.: Global stability of enzymatic chains of full reversible Michaelis-Menten reactions. Acta Biotheoretica 61(3), 425-436 (2013)

6. Belgacem, I., Gouzé, J.L.: Stability analysis and reduction of gene transcription models. In: 2013 IEEE 52nd Annual Conference on Decision and Control (CDC), pp. 2691-2696. IEEE (2013)

7. Belgacem, I., Gouzé, J.L.: Mathematical study of the global dynamics of a concave gene expression model. In: 2014 22nd Mediterranean Conference of Control and Automation (MED), pp. 1341-1346. IEEE (2014) 
8. Belgacem, I., Grac, E., Ropers, D., Gouzé, J.L.: Stability analysis of a reduced transcriptiontranslation model of RNA polymerase. In: 2014 IEEE 53rd Annual Conference on Decision and Control (CDC), pp. 3924-3929. IEEE (2014)

9. Berman, A., Plemmons, R.J.: Nonnegative Matrices in the Mathematical Sciences. SIAM (1994)

10. Bernstein, J.A., Khodursky, A.B., Lin, P.H., Lin-Chao, S., Cohen, S.N.: Global analysis of mRNA decay and abundance in Escherichia coli at single-gene resolution using two-color fluorescent DNA microarrays. Proceedings of the National Academy of Sciences 99(15), 9697-9702 (2002)

11. Berthoumieux, S., de Jong, H., Baptist, G., Pinel, C., Ranquet, C., Ropers, D., Geiselmann, J.: Shared control of gene expression in bacteria by transcription factors and global physiology of the cell. Molecular Systems Biology 9(1) (2013)

12. Bremer, H., Dennis, P.P., et al.: Modulation of chemical composition and other parameters of the cell by growth rate. Escherichia coli and Salmonella: Cellular and Molecular Biology 2(2), 1553-69 (1996)

13. De Leenheer, P., Angeli, D., Sontag, E.: Monotone chemical reaction networks. Journal of Mathematical Chemistry 41(3), 295-314 (2007)

14. Earnest, T.M., Lai, J., Chen, K., Hallock, M.J., Williamson, J.R., Luthey-Schulten, Z.: Toward a whole-cell model of ribosome biogenesis: kinetic modeling of SSU assembly. Biophysical Journal 109(6), 1117-1135 (2015)

15. Heinrich, R., Schuster, S.: The Regulation of Cellular Systems. Springer Science \& Business Media (2012)

16. Hirsch, M.W., Smith, H.L.: Competitive and cooperative systems: a mini-review. In: Positive Systems, pp. 183-190. Springer (2003)

17. Honkela, A., Peltonen, J., Topa, H., Charapitsa, I., Matarese, F., Grote, K., Stunnenberg, H.G., Reid, G., Lawrence, N.D., Rattray, M.: Genome-wide modeling of transcription kinetics reveals patterns of RNA production delays. Proceedings of the National Academy of Sciences 112(42), 13,115-13,120 (2015)

18. Hoppensteadt, F.C.: Singular perturbations on the infinite interval. Transactions of the American Mathematical Society 123(2), 521-535 (1966)

19. Ishihama, A., Fujita, N., Glass, R.E.: Subunit assembly and metabolic stability of E. coli RNA polymerase. Proteins: Structure, Function, and Bioinformatics 2(1), 42-53 (1987)

20. Jacquez, J.A., Simon, C.P.: Qualitative Theory of Compartmental Systems. SIAM Review 35, 43-79 (1993)

21. Keseler, I.M., Mackie, A., Santos-Zavaleta, A., Billington, R., Bonavides-Martínez, C., Caspi, R., Fulcher, C., Gama-Castro, S., Kothari, A., Krummenacker, M., et al.: The EcoCyc database: reflecting new knowledge about Escherichia coli K-12. Nucleic acids research 45(D1), D543-D550 (2017)

22. Khalil, H.: Nonlinear Systems. Prentice Hall (2002)

23. Kremling, A.: Comment on mathematical models which describe transcription and calculate the relationship between mRNA and protein expression ratio. Biotechnology Bioengineering 96(4), 815-819 (2007)

24. Monod, J.: Recherches sur la Croissance des Cultures Bactériennes. Hermann (1942)

25. Raser, J.M., O'shea, E.K.: Noise in gene expression: origins, consequences, and control. Science 309(5743), 2010-2013 (2005)

26. Raveh, A., Margaliot, M., Sontag, E.D., Tuller, T.: A model for competition for ribosomes in the cell. Journal of the Royal Society Interface 13(116), 20151,062 (2016)

27. Sakamoto, K.: Invariant manifolds in singular perturbation problems for ordinary differential equations. Proceedings of the Royal Society of Edinburgh Section A: Mathematics 116(1-2), 45-78 (1990)

28. Shajani, Z., Sykes, M.T., Williamson, J.R.: Assembly of bacterial ribosomes. Annual Review of Biochemistry 80, 501-526 (2011)

29. Smith, H.L.: Cooperative systems of differential equations with concave nonlinearities. Nonlinear Analysis: Theory, Methods \& Applications 10(10), 1037-1052 (1986)

30. Smith, H.L.: On the asymptotic behavior of a class of deterministic models of cooperating species SIAM Journal on Applied Mathematics 46(3), 368-375 (1986)

31. Smith, H.L.: Monotone Dynamical Systems: an Introduction to the Theory of Competitive and Cooperative Systems, vol. 41. American Mathematical Society Mathematical Surveys and Monographs (1995)

32. Sontag, E.: Some new directions in control theory inspired by systems biology. System Biology 1(1), 10 (2004) 
33. Tadigotla, V.R., Maoiléidigh, D.Ó., Sengupta, A.M., Epshtein, V., Ebright, R.H., Nudler, E., Ruckenstein, A.E.: Thermodynamic and kinetic modeling of transcriptional pausing. Proceedings of the National Academy of Sciences 103(12), 4439-4444 (2006)

34. Wada, A.: Growth phase coupled modulation of Escherichia coli ribosomes. Genes to Cells 3(4), 203-208 (1998)

35. Wang, L., Sontag, E.D.: Singularly perturbed monotone systems and an application to double phosphorylation cycles. Journal of Nonlinear Science 18(5), 527-550 (2008)

\section{A Monotone Systems}

Monotone systems form an important class of dynamical systems. They are particularly well adapted to mathematical models in biology ([32]), because they are defined by conditions related to the signs of the Jacobian matrix. Such a sign for one element reflects the fact that some variable will contribute positively to the variation of some other variables, a kind of qualitative dependence frequently found in biological models. The reader may consult the reference [31] for a review and an exhaustive presentation of the theory of monotone systems.

In summary, if the system is cooperative, then the flow preserves the partial order of trajectories in $\Re^{n}$ (the flow is monotone). Consider an autonomous differential system:

$$
\dot{x}=f(x)
$$

where, $x \in \Re^{n}$ and $f: \Re^{n} \rightarrow \Re^{n}$.

The system is monotone if $x_{01} \leq x_{02}$ (this inequality must be understood coordinate by coordinate: i.e. $\left.x_{01 i} \leq x_{02 i}, \forall i \in[1, \ldots, n]\right)$, implies that $x\left(t, x_{01}\right) \leq x\left(t, x_{02}\right) \forall t\left(x\left(t, x_{0}\right)\right.$ corresponds to the evolution with respect to time starting from the initial condition $x_{0}$ ).

Cooperativity is easy to check by looking at the signs of the elements of the Jacobian matrix, which should verify

$$
\frac{\partial f_{i}}{\partial x_{j}}(t, x) \geq 0 \quad \forall i \neq j .
$$

These systems have a strong tendency to converge to the set of their equilibria ([31]). It can be shown that almost any solution converges to the set of equilibria except a set of zero measure. In particular, there are no stable periodic solutions. For more precise theorems, see [31].

\section{B Tikhonov's theorem}

This theorem applies to reduced systems of the form:

$$
\begin{aligned}
& \dot{x}=f(x, y, \varepsilon) \\
& \dot{y}=\frac{1}{\varepsilon} g(x, y, \varepsilon) .
\end{aligned}
$$

where $x \in \Re^{n}, y \in \Re^{m}$, and $0<\varepsilon \ll 1$ ( $\varepsilon$ a very small parameter), $x(0)=x_{0}, y(0)=y_{0}$. So, when $\varepsilon$ tends to $0(\dot{y}$ evolves very rapidly compared to $\dot{x})$, the system (26) is equivalent to the system:

$$
\begin{aligned}
& \dot{x}=f(x, y, 0) \\
& g(x, y, 0)=0
\end{aligned}
$$

This is valid only if the fast subsystem $\dot{y}=g(x, y, 0)$ satisfies some conditions which are given as follows:

- Existence and uniqueness of the steady state (there exists a unique solution, $y^{*}=\phi(x)$ of $g(x, y, 0)=0$.

- Exponential stability of the steady state $y^{*}$ of the fast subsystem $\dot{y}=g(x, y, 0)$ for fixed $x$.

These conditions are given by the Tikhonov's theorem (see [22] for a complete description), which ensures that $y$ will converge rapidly to a quasi-steady state $(y=\phi(x)$, depending only on $x)$. Therefore the reduced system using the Tikhonov's Theorem is:

$$
\dot{x}=f(x, \phi(x), 0), x(0)=x_{0} .
$$

There are also extensions for infinite time [18,22,27]. 


\section{Rescaling the system}

To make the time scales more obvious and verify that the evolution of $z$ and $q$ is slow, we scale the variables $y, y^{1}, \ldots, y^{L-1}$ with respect to a scaling factor $\alpha$, and the variables $x, x^{1}, \ldots, x^{H-1}$ with respect to a scaling factor $\beta$. Consider $\bar{y}=\alpha y, \bar{y}^{i}=\alpha y^{i}$ and $\bar{x}=\beta x, \bar{x}^{i}=\beta x^{i}$, this gives:

$$
\begin{aligned}
\dot{c} & =k_{+} p\left(d_{0}-c\right)-k_{-} c-k_{c} c-k_{p}^{\prime} c \\
\dot{p} & =-k_{+} p\left(d_{0}-c\right)+\frac{k_{t}}{\alpha} \bar{y}^{L-1}+k_{-} c+\frac{k_{t}^{\prime}}{\beta} \bar{x}^{H-1}-k_{p}^{\prime} p \\
\bar{y} & =\alpha k_{c} c-k_{t} \bar{y}-k_{p}^{\prime} \bar{y} \\
\bar{y}^{1} & =k_{t} \bar{y}-k_{t} \bar{y}^{1}-k_{p}^{\prime} \bar{y}^{1} \\
\bar{y}^{2} & =k_{t} \bar{y}^{1}-k_{t} \bar{y}^{2}-k_{p}^{\prime} \bar{y}^{2} \\
\vdots & \\
\bar{y}^{L-1} & =k_{t} \bar{y}^{L-2}-k_{t} \bar{y}^{L-1}-k_{p}^{\prime} \bar{y}^{L-1} \\
\dot{w} & =k_{+}^{\prime} r m-k_{-}^{\prime} w-k_{w} w-k_{m}^{\prime} w \\
\dot{m} & =-k_{+}^{\prime} r m+k_{-}^{\prime} w+k_{w} w+\frac{k_{t}}{\alpha} \bar{y}^{L-1}-k_{m}^{\prime} m \\
\dot{r} & =-k_{+}^{\prime} r m+k_{-}^{\prime} w+\frac{k_{t}^{\prime}}{\beta} \bar{x}^{H-1}+k_{m}^{\prime} w \\
\overline{\dot{x}} & =\beta k_{w} w-k_{t}^{\prime} \bar{x} \\
\dot{x}^{1} & =k_{t}^{\prime} \bar{x}-k_{t}^{\prime} \bar{x}^{1} \\
\vdots & \\
\overline{\dot{x}}^{H-1} & =k_{t}^{\prime} \bar{x}^{H-2}-k_{t}^{\prime} \bar{x}^{H-1}
\end{aligned}
$$

where $\frac{k_{t}}{\alpha}, \frac{k_{t}^{\prime}}{\beta}$ are small compared to $k_{t}$ and $k_{t}^{\prime}$, but where the first one is bigger than the second one $\left(\frac{k_{t}}{\alpha}=200 \mathrm{~min}^{-1} \text { and } \frac{k_{t}^{\prime}}{\beta}=10 \mathrm{~min}^{-1}\right)^{7}$.

Finally the slow evolution part is given by the equation: $z=c+p+\frac{1}{\alpha}\left(\bar{y}+\bar{y}^{1}+\ldots+\bar{y}^{L-1}\right)$, which gives $\dot{z}=\frac{k_{t}^{\prime}}{\beta} \bar{x}^{H-1}-k_{p}^{\prime} z$. Similarly, $q=m+w$ therefore $\dot{q}=\frac{k_{t}}{\alpha} \bar{y}^{L-1}-k_{m}^{\prime} q$. Having introduced the two new variables $z$ and $q$ we return, for simplicity, to the scale of the original system (see system (4)).

7 To obtain these values we choose $\alpha=11.7$ and $\beta=125.8$. 